\title{
CyberKnife Ablation of a Solitary Para-Aortic Lymph Node Metastasis After Robotic-Assisted Radical Prostatectomy
}

Faith Hirri'1, ", Muhammad Arshad Irshad Khalil', Muhammad Shoaib Anwar Bajwa ${ }^{1}$, Naveed Afzal ${ }^{1}$

${ }^{I}$ Department of Urology, Dorset County Hospital NHS Trust, Dorset, United Kingdom

*Corresponding author: Faith Hirri, Department of Urology, Dorset County Hospital NHS Foundation Trust, Williams Ave., Dorchester, Dorset, DT1 2JY,

DOI: $10.30699 / \mathrm{mci} .4 .3 .38$ United Kingdom.Tel: +441305251150;E-mail:faithhirri@gmail.com

Submitted: 30 April 2020

Revised: 26 May 2020

Accepted: 11 June 2020

e-Published: 1 July 2020

\section{Keywords:}

Prostatic Neoplasms

Oligometastases

Stereotactic Body

Radiotherapy

CyberKnife Radiosurgery

Antineoplastic Agents,

Hormonal

\begin{abstract}
Introduction: Radical prostatectomy is an effective curative treatment option for organ-confined prostate cancer. There is a recent trend in offering curative treatment to patients with oligometastatic disease. More sensitive imaging modalities can identify oligometastatic disease that is not usually detected by conventional imaging techniques. Case presentation: We present a case in which a solitary left para-aortic metastatic lesion was ablated; using CyberKnife image-guided stereotactic radiotherapy after robotic-assisted radical prostatectomy with negative margins and negative lymph node status.

Conclusion: With the increasing number of patients diagnosed with oligometastatic prostate cancer, there is a paradigm shift towards its treatment with curative intent. The unusual sites of metastasis, can be cured effectively with Cyber Knife technology, whilst minimising adverse effects. Our report is an effort to highlight this technique as an effective treatment modality to be used and popularised as a standard option.
\end{abstract}

(C) 2020. Multidisciplinary Cancer Investigation

\section{INTRODUCTION}

Radical prostatectomy is an effective curative treatment option for organ-confined prostate cancer [1]. There is a recent trend towards offering curative treatment to patients with an oligometastatic disease where local disease can be treated with surgery or radiotherapy [2]. The usual site of metastasis for prostate cancer is to the regional lymph nodes and bony skeleton [3]. External beam radiotherapy or surgery has been the approach generally offered as the curative treatment option to metastatic lesions in this patient subset $[4,5]$. We present a case in which a solitary left para-aortic metastatic lesion was ablated; using CyberKnife image-guided stereotactic radiotherapy. Although this is not a novel technique, it is not very commonly practiced either. Our report will confirm the utility of this technique in this patient subset who have a highgrade oligometastatic disease and will help in establishing this technique as standard care.

\section{CASE PRESENTATION}

A 69-year-old man was referred to the department of urology after he was found to have an elevated prostate-specific antigen (PSA) level of $5.78 \mathrm{ng}$ / $\mathrm{ml}$, on his routine preventive screening. He had a positive family history. His father was diagnosed with metastatic prostate cancer and had been taking luteinizing hormone-releasing hormone (LHRH) 
analogs injections for the previous 7 years. The digital rectal examination demonstrated a palpable nodule in the left lobe of the prostate. Magnetic resonance imaging (MRI) of the pelvis revealed a $50 \mathrm{cc}$ prostate with a large low T2 defect throughout much of the left peripheral zone, highly suspicious of an important tumor. There was also evidence of significant capsule abutment with no overt T3 disease, or lymphadenopathy (Figure 1).

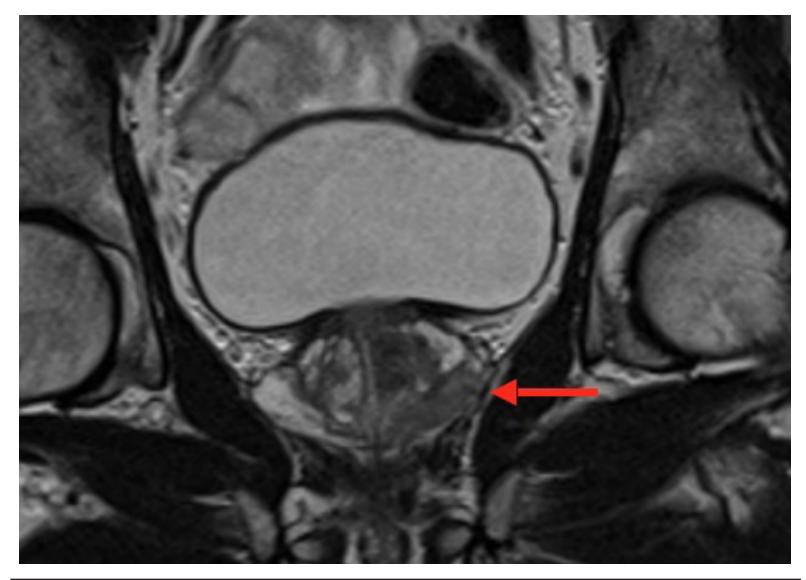

Figure 1: Coronal MRI Pelvis Image, Demonstrating a Large Low T2 Defect Throughout the Left Peripheral Zone With Capsule Abutment (arrow).

A trans-rectal ultrasound (TRUS) and biopsy revealed a Gleason $4+4=8$ adenocarcinoma of the prostate, with all six cores involved on the left side and up to $80 \%$ of core involvement. After a discussion at the multidisciplinary meeting, he underwent roboticassisted radical prostatectomy (RARP) with lymphadenectomy on the left side. Histology confirmed Gleason $4+4=8$ adenocarcinoma with a tertiary pattern five. There was an extraprostatic extension but the resection margins were clear. There were no metastatic deposits in all the five resected lymph nodes (PT3aN0M0). At initial follow-up performed six weeks post-operatively, his PSA level was $1.52 \mathrm{ng} / \mathrm{ml}$ which was unexpected given the complete excision of cancer with negative surgical margins and lymph nodes. A Choline positron emission tomography (PET) scan was subsequently arranged which showed a solitary avid left para-aortic lymph node (Figure 2).

The patient was referred for stereotactic radiotherapy via CyberKnife to the para-aortic lymph node with hormonal therapy; using LHRH analogs for 6 months. The patient completed his hormonal treatment in August 2019 and is still maintaining an undetectable PSA of $<0.02 \mathrm{ng} / \mathrm{ml}$, while his current Testosterone level is within the normal range at $12.1 \mathrm{nmol} / \mathrm{L}$; suggesting complete ablation of the metastatic deposit.

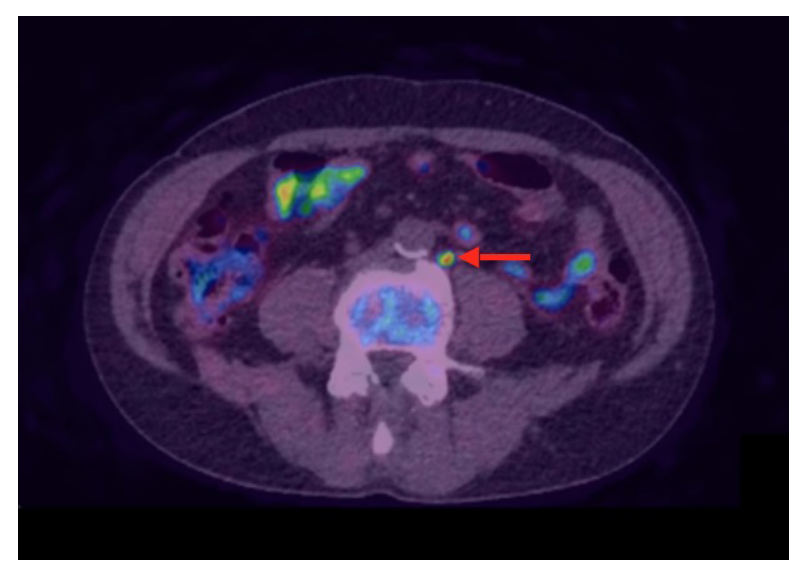

Figure 2: Choline PET Scan Demonstrating an Avid Left ParaAortic Lymph Node (arrow).

\section{DISCUSSION}

The curative management of localized prostate cancer is performed either by radical prostatectomy, brachytherapy, or by external beam radiotherapy, usually with a limited course of androgen deprivation therapy (ADT). With the use of more sensitive hybrid imaging for metastatic workup, a greater number of patients are diagnosed with metastases at sites not usually detected by conventional imaging modalities [4]. Isolated metastasis to the para-aortic lymph nodes is not common [3]. It is a diagnostic challenge and choline or Prostate-specific membrane antigen (PSMA)/PET scan is more valuable in this situation $[4,6]$. This site is difficult to approach surgically and conventional external beam radiotherapy is not without side effects. The novel technique of CyberKnife stereotactic radiotherapy delivers very precise high doses of radiation to the target lesion with minimal damage to the surrounding tissue. After its first use, reported by Jereczek-Fossa et al., this technique has been employed in several series with proven efficacy [3, 7]. Since high-dose radiotherapy can ablate the lesion completely, the need for a prolonged course of hormonal therapy could be delayed or avoided [6]. This improves the patient's tolerability by avoiding surgery as well as reducing the toxicity from radiation therapy and ADT. The net result is an improvement in the quality of life. 
With the increasing number of patients diagnosed with oligometastatic prostate cancer, there is a paradigm shift towards its treatment with curative intent. The unusual sites of metastasis, like para-aortic lymph nodes, could be cured effectively with CyberKnife technology whilst minimizing the adverse effects, thus improving the treatment-related adverse effects [3]. Our report is an effort to highlight this technique as an effective treatment modality to be used and popularized as a standard option, as more literature become available in the future.

\section{ACKNOWLEDGEMENTS}

Not applicable

\section{CONFLICTS OF INTEREST}

The authors declared no conflict of interest.

\section{ETHICS APPROVAL}

Informed consent was obtained from the patient for their anonymized information to be published in this case report

\section{REFERENCES}

1. Abdollah F, Sun M, Schmitges J, Tian Z, Jeldres C, Briganti A, et al. Cancer-specific and other-cause mortality after radical prostatectomy versus observation in patients with prostate cancer: competing-risks analysis of a large North American population-based cohort. Eur Urol. 2011;60(5):920-30. DOI: 10.1016/j.eururo.2011.06.039 PMID: 21741762.

2. Kim J, Park JS, Ham WS. The role of metastasis-directed therapy and local therapy of the primary tumor in the management of oligometastatic prostate cancer. Investig Clin Urol. 2017;58(5):307-16. DOI: 10.4111/icu.2017.58.5.307 PMID: 28868501.

3. Jereczek-Fossa BA, Fariselli L, Beltramo G, Catalano G, Serafini F, Garibaldi C, et al. Linac-based or robotic image-guided stereotactic radiotherapy for isolated lymph node recurrent prostate cancer. Radiother Oncol. 2009;93(1):14-7. DOI: 10.1016/j.radonc.2009.04.001 PMID: 19409636.

4. Cysouw M, Bouman-Wammes E, Hoekstra O, van den Eertwegh A, Piet M, van Moorselaar J, et al. Prognostic Value of [(18)F]-Fluoromethylcholine Positron Emission Tomography/Computed Tomography Before Stereotactic Body Radiation Therapy for Oligometastatic Prostate Cancer. Int J Radiat Oncol Biol Phys. 2018;101(2):406-10. DOI: 10.1016/j.ijrobp.2018.02.005 PMID: 29559285.

5. Gandaglia G, Fossati N, Stabile A, Bandini M, Rigatti P, Montorsi F, et al. Radical Prostatectomy in Men with Oligometastatic Prostate Cancer: Results of a Single-institution Series with Long-term Follow-up. Eur Urol. 2017;72(2):289-92. DOI: 10.1016/j.eururo.2016.08.040 PMID: 27574820.

6. Crehange G, Chen CP, Hsu CC, Kased N, Coakley FV, Kurhanewicz J, et al. Management of prostate cancer patients with lymph node involvement: a rapidly evolving paradigm. Cancer Treat Rev. 2012;38(8):956-67. DOI: 10.1016/j.ctrv.2012.05.005 PMID: 22703831.

7. Decaestecker K, De Meerleer G, Lambert B, Delrue L, Fonteyne V, Claeys T, et al. Repeated stereotactic body radiotherapy for oligometastatic prostate cancer recurrence. Radiat Oncol. 2014;9:135. DOI: 10.1186/1748-717X-9135 PMID: 24920079. 